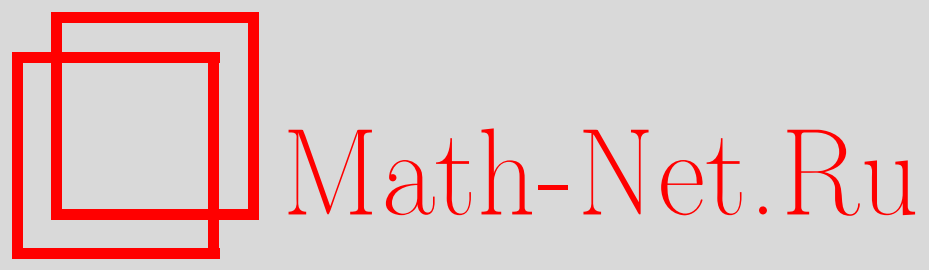

Г. С. Петров, О неколеблемости эллиптических интегралов, Функи. анализ и его прил., 1997, том 31, выпуск 4, 47-51

DOI: https://doi.org/10.4213/faa491

Использование Общероссийского математического портала MathNet.Ru подразумевает, что вы прочитали и согласны с пользовательским соглашением

http://www . mathnet.ru/rus/agreement

Параметры загрузки:

IP: 18.208 .226 .222

26 апреля 2023 г., 07:14:30

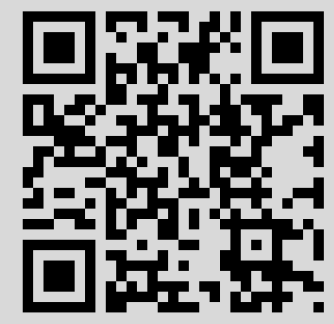


Функииональный анализ и его приложения

1997, т. 31, вып. 4, с. 47-51

УДК 517.583

\title{
О неколеблемости эллиптических интегралов
}

\author{
(c) 1997. Г. С. Петров
}

В. И. Арнольду к его шестидесятилетию

Рассмотрим эллиптический интеграл

$$
\oint P(x, y) d x+Q(x, y) d y
$$

от вещественной полиномиальной 1-формы по вещественным овалам $y^{2}+x^{2}+$ $x^{4}=t$ на плоскости, $t>0$.

В статье дается оценка числа нулей интеграла (1), рассматриваемого как функция от $t$. Оценка опирается на новое утверждение о неколеблемости линейной комбинации собственных функций линейного дифференциального оператора второго порядка (см. лемму 1 ниже).

K задаче об оценке числа нулей интеграла от вещественной полиномиальной формы по овалам уровня вещественного полинома сводится задача об оценке числа предельных циклов, рождающихся при возмущении гамильтонова полиномиального векторного поля.

Обозначим через $\mathscr{J}_{n}$ пространство интегралов (1) от вещественных полиномиальных 1-форм степени не выше $n$.

Основной результат статьи - это следующая

Теорема 1. Пространство $\mathscr{J}_{n}$ лвляется чебышёвским на луче $t>0$. Это означает, что число нулей (с учетом кратности) вслкого интеграла из этого пространства (не равного тождественно нулю) меньше размерности самого пространства. Размерность пространства $\mathscr{J}_{n}$ равна $2[(n-1) / 2]+1$.

Аналог этой теоремы для интегралов по овалам $y^{2}+x^{4}-x^{2}=t$ был доказан ранее автором в работе [1]. Его доказательство применимо и для интегралов из теоремы 1. Здесь приводится другой (вещественный) вариант доказательства.

1. Схема доказательства теоремы 1. Доказательство теоремы 1 основано на следующем утверждении.

Лемма 1. Пусть $f, g$ - непрерывные функиии на интервале $I=(a, b)$ (возможно (полу)бесконечном), причем функция $f$ положительна. Пусть $\lambda_{1}<\cdots<\lambda_{k}$ и существуют решения $y_{1}, \ldots, y_{k}$ уравнений $\ddot{y}_{i}=\left(\lambda_{i} f(t)+\right.$ $g(t)) y_{i}$, обладаюшие следуюшими свойствами:

1) $y_{1}$ нигде не обращается в нуль на $I$;

2) $y_{i}(t) \rightarrow 0$ nри $t \rightarrow a$;

3) производные функиий $y_{i}$ ограничены в окрестности точки а. 
Тогда пространство функчий вида $\sum_{i=1}^{k} c_{i} y_{i}$ имеет размерность $k u$ является чебышёвским.

Лемма 1 доказывается в конце статьи.

Первоначально эта лемма была сформулирована и доказана автором для случая $g=0, f(t)=1 /(t(4 t+1))$. Именно этот частный случай леммы используется при доказательстве теоремы 1 . А. Г. Хованский заметил, что доказательство автора полностью остается в силе в условиях леммы 1 в общем случае. Отметим, что при $k=2$ в предположении, что функции $f$ и $g$ непрерывно продолжаются в точку $a$, лемма 1 является частным случаем теоремы сравнения Штурма (см. ниже п. 4).

Мы покажем, что пространство $\mathscr{J}_{n}$ принадлежит классу чебышёвских пространств из леммы 1.

Справедливость теоремы 1 вытекает из леммы 1 и следующих трех утверждений.

Лемма 2. В пространстве $\mathscr{J}_{n}$ имеется базис $\left\{y_{i}\right\}$, состояший из решений уравнений второго порядка

$$
\ddot{y}_{i}=\frac{\lambda_{i}}{t(4 t+1)} y_{i}, \quad \lambda_{1}<0<\lambda_{2}<\ldots .
$$

Решение $y_{1}$ положительно всюду.

Лемма 2 доказывается в следующем пункте.

Лемма 3. Пространство $\mathscr{J}_{n}$ - это в точности пространство полиномиальньх линейньх комбинаций

$$
I_{0} P_{0}+I_{1} P_{1}
$$

специальньх эллиптических интегралов $I_{0}$ и $I_{1}$ от форм $\omega_{0}=y d x u \omega_{1}=$ $x^{2} y d x$ с вещественными полиномами $P_{0}$ и $P_{1}$ степеней не выше $[(n-1) / 2]$ $u[(n-1) / 2]-1$ соответственно.

ПРЕДЛОЖЕНИЕ 1. Все интеграль (1) аналитически продолжаются нулем в точку $t=0$.

Аналоги леммы 3 и предложения 1 для интегралов по овалам $y^{2}+x^{4}-x^{2}=t$ содержатся в [1]. Доказательство применимо и для интегралов из пространства $\mathscr{J}_{n}$.

Для доказательства теоремы 1 осталось заметить, что базисные функции $y_{i}$ из леммы 2 удовлетворяют условиям леммы 1 (лемма 2 и предложение 1). В силу леммы 1 пространство $\mathscr{J}_{n}$ является чебышёвским. Утверждение теоремы 1 о размерности этого пространства следует из леммы 3.

2. Доказательство леммы 2. Мы ищем базис $\left\{y_{i}\right\}$ в пространстве $\mathscr{J}_{n}$, удовлетворяющий утверждениям леммы 2. Уравнения на $y_{i}$ означают, что они суть собственные функции оператора $L=t(4 t+1) d^{2} / d t^{2}$.

Справедливость леммы 2 вытекает из следующей леммы.

Лемма 4. Пространство $\mathscr{J}_{n}$ инвариантно относительно оператора $L$. Функиия $I_{0}$ является его собственной функцией с отричательным собственным значением. Остальнье собственные значения оператора L на пространстве $\mathscr{J}_{n}$ положительны и попарно различны. 
Лемма 4 доказывается в п. 3.

Искомый базис $\left\{y_{i}\right\}$ есть собственный базис оператора $L$ с $y_{1}=I_{0}$. Его существование следует из того, что собственные значения этого оператора попарно различны. Для завершения доказательства леммы 2 осталось заметить, что функция $I_{0}(t)$ положительна, так как равна площади области, ограниченной овалом уровня $t$. Лемма 2 доказана.

3. Доказательство леммы 4. Мы покажем, что в базисе из функций $I_{0}, I_{1}, t I_{0}, t I_{1}, \ldots$ оператор $L$ задается верхнетреугольной матрицей, у которой первый диагональный элемент отрицателен, а остальные положительны и попарно различны. Это докажет лемму 4: диагональные элементы верхнетреугольной матрицы суть ее собственные значения, а первая функция $I_{0}$ собственная.

При доказательстве утверждения из предыдущего абзаца будем использовать следующее свойство интегралов $I_{0}, I_{1}$.

ЛЕмМА 5.

$$
I_{0}=\frac{4}{3} t I_{0}^{\prime}-\frac{2}{3} I_{1}^{\prime}, \quad I_{1}=-\frac{2}{15} t I_{0}^{\prime}+\left(\frac{4}{5} t+\frac{4}{15}\right) I_{1}^{\prime} .
$$

Аналогичные уравнения для интегралов по овалам $y^{2}+x^{4}-x^{2}=t$ содержатся в [1]. Они остаются в силе, когда интегралы берутся по произвольному семейству овалов на комплексных поверхностях уровня (например, по овалам, являющимся пересечением поверхности уровня с плоскостью $y \in \mathbb{R}, x \in i \mathbb{R})$. Уравнения леммы 5 получаются из этих уравнений после подстановки $x=i x^{\prime}$.

Покажем вначале, что оператор $L$ сохраняет пространство линейных комбинаций интегралов $I_{0}, I_{1}$ и в базисе $I_{0}, I_{1}$ задается матрицей

$$
\left(\begin{array}{cc}
-3 / 4 & 1 / 2 \\
0 & 5 / 4
\end{array}\right)
$$

Доказательство состоит в прямом вычислении значений оператора $L$ на $I_{0}$ и $I_{1}$. Дифференцируя уравнения (3) и перенося члены, содержащие $I_{j}^{\prime}, j=0,1$, в левую часть, получаем

$$
I_{0}^{\prime}=-4 t I_{0}^{\prime \prime}+2 I_{1}^{\prime \prime}, \quad \frac{2}{15} I_{0}^{\prime}+\frac{1}{5} I_{1}^{\prime}=-\frac{2}{15} t I_{0}^{\prime \prime}+\left(\frac{4}{5} t+\frac{4}{15}\right) I_{1}^{\prime \prime} .
$$

Подставляя правую часть первого уравнения вместо $I_{0}^{\prime}$ во второе, получаем

$$
I_{1}^{\prime}=2 t I_{0}^{\prime \prime}+4 t I_{1}^{\prime \prime}
$$

Теперь выразим $I_{0}, I_{1}$ через их вторые производные. Для этого подставим формулы (5) и (6) для $I_{0}^{\prime}, I_{1}^{\prime}$ в уравнения (3). Имеем

$$
\begin{aligned}
& I_{0}=-\frac{4}{3} t(4 t+1) I_{0}^{\prime \prime}=-\frac{4}{3} L I_{0} \\
& I_{1}=\frac{8}{15} t(4 t+1) I_{0}^{\prime \prime}+\frac{4}{5} t(4 t+1) I_{1}^{\prime \prime}=\frac{8}{15} L I_{0}+\frac{4}{5} L I_{1} .
\end{aligned}
$$

Отсюда следует доказываемое утверждение о действии $L$ на $I_{0}$ и $I_{1}$. 
Теперь мы покажем, что $L$ сохраняет флаг подпространств, отвечающий базису $I_{0}, I_{1}, t I_{0}, t I_{1}, \ldots$ (и, таким образом, соответствующая матрица верхнетреугольная). Мы покажем, что диагональный элемент, отвечающий базисной функции $t^{k} I_{0}$ (функции $t^{k} I_{1}$ ) равен числу $-3 / 4+k(4 k+2)$ (соответственно числу $5 / 4+k(4 k+6))$. Все эти числа положительны за исключением $-3 / 4$ и, как легко проверить, попарно различны. Это докажет утверждение из начала п. 3.

Для доказательства вычислим значение оператора $L$ на функции $t^{k} I_{0}$ (Функции $\left.t^{k} I_{1}\right)$ с точностью до линейных комбинаций предшествующих ей базисных функций. Имеем

$$
L\left(t^{k} I_{j}\right)=t^{k} L I_{j}+k(k-1) t(4 t+1) t^{k-2} I_{j}+2 k t^{k}(4 t+1) I_{j}^{\prime} .
$$

Проведем вычисление для $j=0$. Сумма двух первых членов правой части в $(7)$ равна (с указанной точностью) $(-3 / 4+4 k(k-1))\left(t^{k} I_{0}\right)$ (см. (4)). Для вычисления последнего члена выразим $t(4 t+1) I_{0}^{\prime}$ через $I_{0}$ и $I_{1}$, пользуясь первым из уравнений (5). Домножая это уравнение на $t(4 t+1)$, получаем $t(4 t+1) I_{0}^{\prime}=$ $-4 t L I_{0}+2 L I_{1}$. Это равно $3 t I_{0}$ по модулю линейной комбинации интегралов $I_{0}, I_{1}$ (см. (4)). Отсюда следует, что с точностью, указанной в начале абзаца, последний член правой части (7) равен $6 k t^{k} I_{0}$, а значит, $L\left(t^{k} I_{0}\right)$ равно $(-3 / 4+k(4 k+2)) t^{k} I_{0}$. Аналогично доказывается, что, с соответствующей точностью, $L\left(t^{k} I_{1}\right)$ равно $(5 / 4+k(4 k+6)) t^{k} I_{1}$. Это доказывает утверждения из предыдущего абзаца. Лемма 4 доказана.

4. Доказательство леммы 1. Прежде всего покажем, что ни одна из собственных функций $y_{i}$ нигде не обрашается в нуль на $I$. Для $i=1$ это одно из условий леммы. Для $i>1$ это вытекает из неравенства $\lambda_{i}>\lambda_{1}$ и следующего варианта теоремы сравнения Штурма, примененного к $y_{1}, y_{i}$.

ТеОрема СРАвненИЯ Штурма. Пусть $F(t)<G(t)$ - непрерьвные функиии на интервале $I=(a, b)$ (возможно, (полу)бесконечном). Пусть $y_{1}, y_{2}$ - решения уравнений $\ddot{y}_{1}=F(t) y_{1}, \ddot{y}_{2}=G(t) y_{2}$, не равные тождественно нулю. Пусть функчии $y_{i}$ непрерывно продолжаются нулем в точку а, а их производные ограничены в ее окрестности. Тогда между любыми двумя нулями функиии $y_{2}$ на $I \cup$ а лежит хотя бы один нуль функции $y_{1}$.

Поскольку наш вариант теоремы сравнения Штурма чуть более общий, чем классический вариант [2], приведем полностью его доказательство.

ДокАЗАтЕльство. Предположим, что имеется пара нулей $c, d \in I \cup\{a\}$ функции $y_{2}$, на интервале между которыми $y_{1}$ не обрашается в нуль. Без ограничения общности можно считать, что $y_{1}, y_{2}>0$ на $(c, d)$. Пусть $W=y_{1} \dot{y}_{2}-y_{2} \dot{y}_{1}$ - определитель Вронского пары функций $y_{1}, y_{2}$. С одной стороны, на $(c, d)$ вронскиан $W(t)$ строго возрастает: $\dot{W}=y_{1} \ddot{y}_{2}-y_{2} \ddot{y}_{1}=(G-F) y_{1} y_{2}>0$. C другой стороны, как показывается ниже, $W(c) \geqslant 0 \geqslant W(d)$. (Мы считаем, что $W(a)=0$ : функция $W$ непрерывно продолжается нулем в точку $a$ в силу условия теоремы.) Полученное противоречие доказывает теорему.

Осталось лишь проверить неравенства из конца предыдущего абзаца. По предположению $y_{2}=0$ в точках $c$ и $d$, и, таким образом, $W=y_{1} \dot{y}_{2}$. Функция $y_{1}$ положительна на интервале $(c, d)$, а значит, ее значения в его концах неотрицательны. Это означает, что $W$ принимает в каждой из них либо нулевое 
значение, либо значения того же знака, что и $\dot{y}_{2} \cdot($ Если $c=a$, то $W(c)=0$. Имеем $\dot{y}_{2}(d)<0$, а при $c \in I$ выполняется неравенство $\dot{y}_{2}(c)>0$. Это следует из того, что $\left.y_{2}\right|_{(c, d)}>0$ по предположению. Этим устанавливаются неравенства из предыдущего абзаца. Теорема Штурма доказана.

Для гладкой на интервале $I$ функции $q$ через $\left.\# q\right|_{I}$ будем обозначать число ее нулей, посчитанных с учетом кратности.

Лемма 6. 1. Для всякой гладкой функиии q на интервале I справедливо неравенство $\left.\# q\right|_{I} \leqslant\left. \# q^{\prime}\right|_{I}+1$.

2. Если функиия $q$ стремится $\kappa$ нулю при стремлении аргумента к одному из концов интервала $I$, то справедливо неравенство \#q $\left.\right|_{I} \leqslant\left. \# q^{\prime}\right|_{I}$.

Лемма 6 следует из теоремы Ролля: между любыми двумя нулями функции лежит хотя бы один нуль производной.

Вернемся к доказательству леммы 1. Пусть $Y=\sum_{i \leqslant k} c_{i} y_{i}$ - нетривиальная линейная комбинация функций $y_{i}$. Обозначим через $q$ частное

$$
\frac{Y}{y_{k}}=c_{k}+\sum_{i \leqslant k} c_{i} \frac{y_{i}}{y_{k}} .
$$

Нам надо доказать, что $\left.\# q\right|_{I} \leqslant k$. В силу п. 1 из леммы 6 достаточно показать, что $\left.\# q^{\prime}\right|_{I}<k-1$. Имеем

$$
q^{\prime}=\frac{h}{y_{k}^{2}}, \quad \text { где } h=\sum_{i<k} c_{i}\left(y_{i}^{\prime} y_{j}-y_{i} y_{j}^{\prime}\right) .
$$

Таким образом, достаточно доказать аналогичную оценку для \# $\left.\right|_{I}$. Функция $h$ непрерывно продолжается нулем в точку $a$ (ограниченность производных $y_{i}^{\prime}$ ). Поэтому, согласно п. 2 леммы 6,

$$
\left.\# h\right|_{I} \leqslant\left. \# h^{\prime}\right|_{I}
$$

Далее,

$$
h^{\prime}=\sum_{i<k} c_{i}\left(y_{i}^{\prime \prime} y_{j}-y_{i} y_{j}^{\prime \prime}\right)=y_{j} f \sum_{i<k} c_{i}\left(\lambda_{i}-\lambda_{j}\right) y_{i} .
$$

Сумма в правой части последнего равенства есть линейная комбинация $k-1$ функций $y_{i}, i<k$. По индукции она либо тождественно равна нулю, либо имеет менее $k-1$ нулей. Лемма 1 доказана.

Я благодарен Ю. С. Ильяшенко и А. Г. Хованскому за полезные замечания, а также А. А. Глуцюку за помощь при подготовке статьи к печати.

\section{ЛИТЕРАТУРА}

1. Петров Г. С. Эллиптические интегралы и их неколеблемость. Функц. анализ и его прил., 20, вып. 1, 46-49 (1986).

2. Степанов B. В. Курс дифференциальных уравнений. Изд. 7-е, М., 1958. 